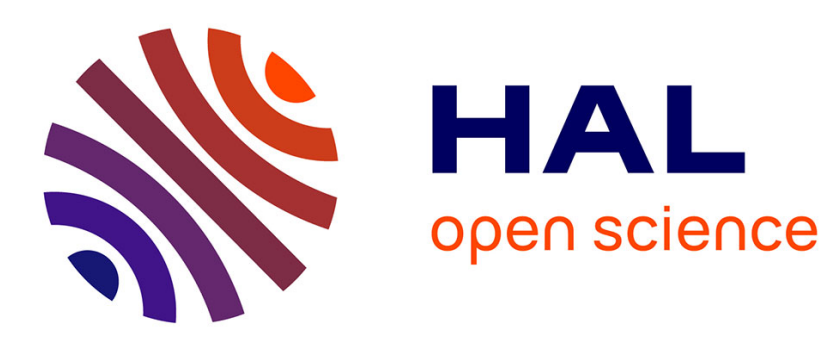

\title{
Influence des non-linéarités sur les caractéristiques des oscillations des instruments à vent
}

\author{
Noël Grand, Joel Gilbert, F. Laloë
}

\section{To cite this version:}

Noël Grand, Joel Gilbert, F. Laloë. Influence des non-linéarités sur les caractéristiques des oscillations des instruments à vent. Journal de Physique IV Proceedings, 1994, 04 (C5), pp.C5-585-C5-588. 10.1051/jp4:19945124. jpa-00252800

\section{HAL Id: jpa-00252800 https://hal.science/jpa-00252800}

Submitted on 1 Jan 1994

HAL is a multi-disciplinary open access archive for the deposit and dissemination of scientific research documents, whether they are published or not. The documents may come from teaching and research institutions in France or abroad, or from public or private research centers.
L'archive ouverte pluridisciplinaire HAL, est destinée au dépôt et à la diffusion de documents scientifiques de niveau recherche, publiés ou non, émanant des établissements d'enseignement et de recherche français ou étrangers, des laboratoires publics ou privés. 


\title{
Influence des non-linéarités sur les caractéristiques des oscillations des instruments à vent
}

\author{
N. GRAND*, J. GILBERT** et F. LALOË
}

\author{
* LOA/ESPCI, Univ. Paris 7, URA 1503 du CNRS, 10 rue Vauquelin, 75005 Paris, France \\ ** Laboratoire d'Acoustique de l'Université du Maine, URA 1101 du CNRS, avenue Olivier Messiaen, \\ BP. 535, 72017 Le Mans cedex, France
}

\begin{abstract}
Two simple equations give the minimal modelisation of the acoustical behaviour of woodwind instruments. We discuss the predictions of these equations on the characteristics of the small oscillations, in particular their quasisinusoidal character or finite harmonic content, and domain of existence. We show that, instead of predicting a unique and simple standard behaviour, they introduce a whole series of possible cases (including normal and inverse bifurcations), depending on the characteristics of the non linearity and of the resonator.
\end{abstract}

\section{Introduction: modèle minimal}

Le modèle à non linéarités parfaitement localisées est probablement celui qui permet la description la plus simple d'un instrument à vent. Dans ce dernier on distingue deux éléments: le résonateur lui même, qui fonctionne en régime linéaire mais qui, du fait de la propagation des ondes acoustiques en son sein, introduit des retards; l'excitateur non linéaire qui injecte l'énergie dans le résonateur. Ce dernier peut être régi par une équation non linéaire compliquée, mais n'introduit aucun retard. Si l'on note $p(t)$ la pression acoustique dans le résonateur au point où il est couplé à l'excitateur, et $f(t)$ le débit acoustique en ce point, les équations correspondant à ce "modèle minimal" s'écrivent $[1],[2],[3],[4]:$

$$
\left\{\begin{array}{l}
\bar{p}(\omega)=Z(\omega) \bar{f}(\omega) \\
f(t)=F[p(t)]
\end{array}\right.
$$

où $\bar{p}(\omega)$ est la transformée de Fourier de $p(t), Z(\omega)$ l'impédance acoustique du résonateur et $F$ la fonction non linéaire donnant le débit en fonction de la pression. Dans le domaine temporel, la première équation se réécrit instantanément comme une intégrale de convolution faisant intervenir $p(t)$ et la transformée de Fourier inverse ${ }^{1} G(t)$ de $Z(\omega)$. Dans l'étude qui suit, nous ne ferons aucune hypothèse restrictive sur la forme de la fonction $F$, si ce n'est qu'elle est suffisamment régulière pour être développable en série de Taylor:

$$
F\left(p_{o}+p\right)=F_{0}+A p+B p^{2}+C p^{3}+\ldots .
$$

Ici, $p_{0}$ est par exemple la pression associée au point de fonctionnement du système en l'absence d'oscillation (avant que le seuil d'oscillation ne soit franchi) et $F_{0}=F\left(p_{0}\right)$; cette quantité est obtenue par intersection de la courbe représentant la fonction $F$ et de la droite d'équation $p=Z_{0} f$ et de pente $1 / Z_{0}$ passant par l'origine. Le paramètre $A$, qui donne la pente de la courbe, joue le rôle de paramètre de contrôle; s'il est nul, l'excitateur ne fournit aucun débit acoustique pour de petites variations de la pression interne au résonateur, et donc aucune énergie: il est clair qu'aucune oscillation ne peut se produire. Que se passe-t-il lorsque l'on fait crôtre ce paramètre? II serait naturel de s'attendre à un scénario très simple où, à partir d'une certaine valeur de seuil pour $A$, des oscillations infiniment

\footnotetext{
${ }^{1} G(t)$ est la réponse impulsionnelle du résonateur.
} 
petites et quasi-sinusoidales apparaissent, pour croitre ensuite avec $A$ et acquérir des composantes de Fourier de plus en plus significatives. De fait, ce scénario est tellement naturel que sa validité semble avoir été admise implicitement dans la littérature. Mais nous allons voir qu'il est loin d'être évident, et que bien d'autres cas peuvent se produire: par exemple, partant de zéro, le niveau d'oscillation peut sauter à une valeur finie, et/ou les petites oscillations peuvent ne pas être quasi-sinusoïdales et avoir un contenu harmonique fini.

Le plan de ce texte est le suivant: en premier lieu, nous étudions mathématiquement les propriétés des petites oscillations quasi-sinusoïdales qui sont solutions du système (1), ainsi que les déplacements en fréquence associés à ces oscillations. Nous établissons ensuite les relations entre les coefficients $A$, $B, C$, etc. du développement de $F$ qui permettent à de telles oscillations d'exister. Enfin, nous examinons un certain nombre de cas particuliers et nous faisons le lien avec des études précédentes comme celles de Maganza et coll. [6] de façon à dégager les lignes générales qui permettent de comprendre quels types d'oscillations sont prévues par le système (1). Pour des raisons de limitation d'espace, le présent texte ne fait qu'esquisser le sujet; un article plus détaillé est en cours de rédaction [5].

\section{Caractéristiques des petites oscillations}

Introduisons les coefficients de Fourier $x_{n}$ de $p(t)=\sum x_{n} e^{i n \omega t}$; le système (1), avec la fonction non-linéaire (2), peut alors être ré-écrit sous la forme d'une infinité d'équations[2]:

$$
\left\{\begin{aligned}
\left(A_{1}-A\right) x_{1}= & 2 B\left[x_{2} x_{1}^{*}+x_{3} x_{2}^{*}+\ldots\right] \\
& +3 C\left[\left|x_{1}\right|^{2} x_{1}+2\left|x_{2}\right|^{2} x_{1}+2 x_{0} x_{1}^{*} x_{2}+2 x_{0} x_{2}^{*} x_{3}+\ldots\right]+\ldots \\
\left(A_{2}-A\right) x_{2}= & {\left[B+3 C x_{0}\right] x_{1}^{2}+2 B\left[x_{3} x_{1}^{*}+\ldots\right] } \\
& +3 C\left[2\left|x_{1}\right|^{2} x_{2}+\left|x_{2}\right|^{2} x_{2}+2 x_{0} x_{1}^{*} x_{3}+\ldots\right]+. . \\
\left(A_{3}-A\right) x_{3}= & 2 B\left[x_{2} x_{1}+\ldots\right]+C x_{1}^{3}+3 C\left[x_{2}^{2} x_{1}^{*}+2 x_{0} x_{1} x_{2}+\ldots\right]+\ldots \\
\ldots & \ldots
\end{aligned}\right.
$$

Dans ce système, les admittances $A_{n}$ sont en général complexes, mais les coefficients $A, B, C$, etc. sont réels. Supposons maintenant que $x_{1}$ soit un infiniment petit ainsi que tous les autres $x_{n}$ (supposés d'ordre égal ou supérieur). La première équation montre alors immédiatement que la quantité $\left(A_{1}-A\right)$ doit nécessairement être également infiniment petite: l'oscillation ne peut apparaitre que lorsque la pente $A$ est égale à l'admittance $A_{1}$ du résonateur, qui est donc nécessairement réelle; l'équation $\operatorname{Im}\left\{A_{1}\left(\omega_{s}\right)\right\}=0$ donne ainsi les fréquences d'oscillation possibles juste au seuil. Supposons maintenant que nous nous trouvions dans le cas général où, pour cette fréquence, $A_{2} \neq A_{1}$; la deuxième équation de ce système implique alors que $x_{2}$ est d'ordre 2 en $x_{1}$. De même, d'après la troisième équation, $x_{3}$ est d'ordre 3 en $x_{1}$ si $A_{2} \neq A_{1}$, et ainsi de suite. Dans le cas général il existe donc des petites oscillations quasi-sinusoïdales, dont le contenu harmonique décroit avec l'ordre, mais il nous reste à déterminer le domaine de ces petites oscillations. En effectuant une résolution des équations jusqu'à l'ordre trois en $x_{1}$, on constate que seules les deux premières équations interviennent, et que seuls les termes d'ordres 3 et inférieurs de la fonction non-linéaire ont un rôle à jouer. Après calcul, il vient:

$$
\left|x_{1}\right|^{2}=\frac{\left(A_{1}-A^{\prime}\right)\left(A_{2}-A^{\prime}\right)}{2\left(B^{\prime}\right)^{2}+3 C\left(A_{2}-A^{\prime}\right)}
$$

avec les notations:

$$
A^{\prime}=A+2 B x_{0}+3 C x_{0}^{2} \quad B^{\prime}=B+3 C x_{0}
$$

L'équation (4) impose comme condition d'existence des petites oscillations que le second membre soit réel et positif. Exploitant cette condition, on peut montrer que les différents types de bifurcations possibles, qui dépendent des paramètres intervenant dans (4), sont ceux représentés sur la figure 1. Dans les refs. [1], [2] et [3], où l'on étudie un résonateur cylindrique de grande admittance pour le second harmonique, et où les études expérimentales sur les variations du débit en fonction de la 


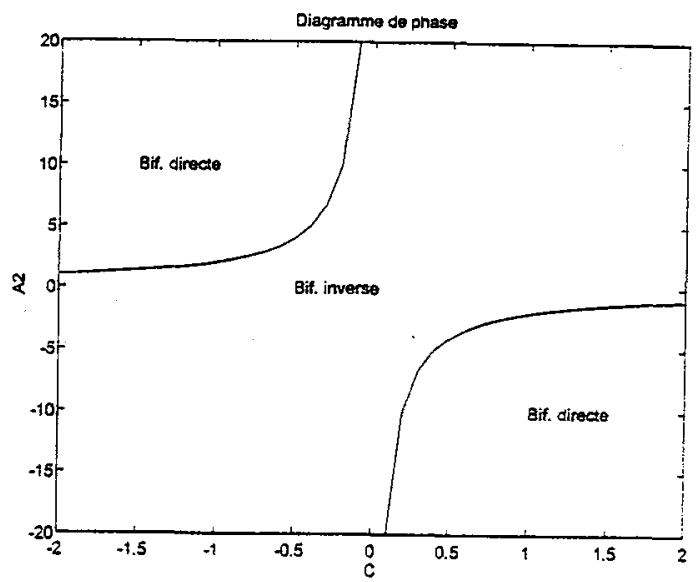

Figure 1: diagramme de phase en fonction de $\mathrm{C}$ (composante cubique de $\mathrm{F}$ ) et la partie reelle de l'admittance pour le second harmonique.

pression (en régime continu) poussent à prendre $C<0$, on tombe dans un cas de bifurcation directe, comme déja noté par Maganza et coll. [6].

La même condition (il suffit en fait d'utiliser la réalité de l'expression (4))permet de calculer la fréquence des petites oscillations quasi-sinusoïdales; supposant que $\omega=\omega_{s}+\delta \omega$ est proche d'une valeur de la fréquence $\omega_{s}$ pour laquelle $A_{1}$ est réel, et appelant $Q$ le facteur de qualité de la résonance correspondante pour l'impédance, nous posons:

$$
A_{1}(\omega)=A_{1}^{s}\left(1+i Q \frac{\delta \omega}{\omega_{s}}\right)+O\left(\delta \omega^{2}\right) ; A_{2}=A_{2 r}^{s}+i A_{2 i}^{s}+O(\delta \omega) ; \quad A=A_{1}^{s}+\delta A
$$

et nous aboutissons à l'expression approchée suivante du déplacement de fréquence:

$$
\frac{\delta \omega}{\omega_{s}} \simeq \frac{\delta A}{Q A_{1}^{s}} \times \frac{2\left(B^{\prime}\right)^{2} A_{2 i}^{s}}{3 C\left(A_{2 i}^{s}\right)^{2}+\left(A_{2}^{s}-A_{1}^{s}\right)\left[2\left(B^{\prime}\right)^{2}-3 C\left(A_{2 r}^{s}-A_{1}^{s}\right)\right]}
$$

\section{Cas particuliers}

\section{1 résonateur en peigne parfait}

Reprenons le cas particulier de résonateur étudié par Maganza et coll. [6], où les résonances d'admittance ou d'impédance forment un peigne parfait et satisfont, pour une fréquence correspondant à la moitié de celle d'aller et retour des ondes sonores dans le tube cylindrique:

$$
\left\{\begin{array}{l}
A_{2 n+1}=\varepsilon \\
A_{2 n}=1 / \varepsilon
\end{array}\right.
$$

Si l'on suppose que les $x_{n}$ sont les coefficients de Fourier d'un signal carré, on peut alors montrer que toutes les équations (3) se dégénèrent en deux sous systèmes, l'un pour les harmoniques pairs qui se réduit à une identité et l'autre pour les harmoniques impairs qui se réduit à une seule équation:

$$
a^{2}=\frac{\varepsilon-A^{\prime}}{C}
$$

où $2 a$ représente l'amplitude crête à crête du signal carré en question. Suivant le signe de $C$, les petites oscillations carrées donnent donc lieu à une bifurcation soit directe $(C<0)$, soit inverse 
$(C>0)$. Nous retrouvons ainsi les résultats de Maganza et coll., obtenus par une autre méthode, qui montrent que c'est le terme cubique de la fonction non linéaire qui commande le domaine d'existence des petites oscillations carrées. C'est un premier cas particulier pour lequel il existe des petites oscillations stables non quasi-sinusoïdales.

\section{2 un cas dégénéré}

Nous avons étudié deux cas limite: celui où la pente $A$ devient égale, soit à l'une seule des admittances ( $A_{1}$ en l'occurrence), soit simultanément à toutes les admittances des harmoniques impairs; le premier est plus général, tandis que le second suppose des propriétés très particulières du résonateur. Nous pouvons également étudier un cas intermédiaire où deux admittances, $A_{1}$ et $A_{2}$ par exemple, sont égales (dégénérescence partielle), ce qui permet à $A$ d'atteindre simultanément les deux valeurs. Dans une telle situation, $x_{1}$ et $x_{2}$ sont des infiniments petits du même ordre, et le calcul du $\S 2$ doit être modifié en conséquence; le calcul doit être poussé plus loin pour inclure les effets de $x_{3}$ et de $x_{4}$ (qui jouent maintenant le rôle précédemment joué par $x_{2}$ ) et l'on obtient une oscillation "quasi bisinusoidale", stable ou instable selon le signe d'une quantité qui dépend de $C, A_{3}$ et $A_{4}$. De façon analogue, on trouverait pour des dégénérescences plus élevées du résonateur des régimes en oscillations "quasi $n$ sinusoidales" jusqu'à la limite $n=\infty$ où l'on retomberait sur le cas du $§ 3.1$.

\section{Conclusion}

Les systèmes d'équations (1) et (3) peuvent être exploités dans bien d'autres cas; par exemple un cas intéressant est celui où le résonateur est supposé ne posséder que deux pics d'impédance, les $Z_{n}$ étant tous strictement nuls à partir du troisième harmonique et au delà. On peut alors non seulement retrouver dans ce cas particulier les résultats plus généraux du $\S 2$, mais aussi étudier les oscillations d'amplitude finie (en supposant toutefois le résonateur parfaitement harmonique). Ceci permet la construction complète d'un "diagramme de phase" donnant les caractéristiques des oscillations d'amplitudes non évanescentes.

La dynamique de l'anche permet également d'introduire une généralisation intéressante. Dans beaucoup de cas, on retombe en fait sur des équations du même type que (1), en réinterprétant toutefois le sens physique des grandeurs qui y figurent (en particulier $Z(\omega)$ n'est plus l'impédance du résonateur mais dépend des caractéristiques dynamiques de l'anche); cela permet d'appliquer directement, presque sans changement, toutes les considérations qui précèdent. Il existe toutefois des cas où la généralisation est moins triviale. Toutes ces situations seront discutées en détail dans une publication future [5].

\section{References}

[1] J. Backus, J. Acoust. Soc. Amer.35, 305 (1963).

[2] W.E. Worman, "Self sustained oscillations in clarinet like systems", thesis, Case Western University (1971).

[3] T.A. Wilson and G.S. Beavers, J. Acoust. Soc. Amer. 56, 653 (1974).

[4] M.E. McIntyre, R.T. Schumacher and J. Woodhouse, J. Acoust. Soc. Amer. 74, 1325 (1983).

[5] N. Grand, J. Gilbert et F. Laloë, en préparation.

[6] C. Maganza, R. Caussé et F. Laloë, Europhysics Letters, 1, 295 (1986). 